\title{
Cyclic Polyamide Oligomers Extracted from Nylon 66 Membrane Filter Disks as a Source of Contamination in Liquid Chromatography/Mass Spectrometry
}

\author{
John C. Tran and Alan A. Doucette \\ Department of Chemistry, Dalhousie University, Halifax, Nova Scotia, Canada
}

\begin{abstract}
Background interferences are perhaps an unavoidable part of analytical detection schemes, particularly when analyzing trace level samples or when using detectors with high levels of sensitivity. In liquid chromatography, mobile phase solvents are routinely filtered using membrane filter disks to trap particulates in hopes of minimizing contamination, providing improvements in data output and instrumental operation. In this study, we report that one such filter disk leads to a significant level of contamination in LC and LC/MS experiments. Extractable compounds from nylon membrane filters generate significant background signals in UV absorption chromatograms at $214 \mathrm{~nm}$, and are also detected by electrospray ionization mass spectrometry, with nominal $\mathrm{m} / z$ values of 453 and 679. It is shown that rinsing the nylon membranes before their use can reduce, but will not eliminate, the extractable contaminants from the mobile phase. Through MS and tandem MS analysis, we have identified these contaminants as cyclic oligomers of polyamide 66. Based on these results, it is recommended that nylon membrane filters be avoided when conducting trace level analysis, particularly when conducting LC/MS experiments. (J Am Soc Mass Spectrom 2006, 17, 652-656) (C) 2006 American Society for Mass Spectrometry
\end{abstract}

$\mathrm{T}$ The availability of exceedingly sensitive detectors has facilitated our ability to perform trace level analyses. Mass spectrometers are prime examples of detectors with marked gains in sensitivity. However, with increased sensitivity comes a growing concern of observing interferences, including those that are introduced during sample workup stages, such as liquid chromatographic separations. Although difficult to completely eliminate, contaminants can be minimized if a sufficient level of care is taken to avoid introducing impurities during sample workup. While common sense can be employed to minimize contaminants, many potential sources are not always evident. Likely the best way to eliminate a particular interference is through knowledge of its origin.

A recent review by Williams provides an excellent account of some common sources of contaminants occurring in reversed-phase gradient LC [1]. Organic compounds present in the weaker solvent (Solvent A) are of particular concern as they tend to concentrate on the column during equilibration and elute during the gradient, giving rise to "ghost peaks" in a chromato-

Published online March 6, 2006

Address reprint requests to Dr. A. A. Doucette, Department of Chemistry, Dalhousie University, 6274 Coburg Road, Halifax, Nova Scotia B3H 4J3, Canada. E-mail: Alan.Doucette@dal.ca gram [2]. Using the highest-purity water available for LC is always a prudent practice [3]. Improperly cleaned glassware is often associated with anomalous results in LC separations; soap residues have also been associated with ghost peaks in a chromatogram [4]. Plastics are also a common source of contamination, and interferences resulting from various extractables and plasticizers have been the subject of various reports [5-7].

In mass spectrometry, an elevated background, often associated with chemical noise, has the potential to seriously reduce the performance of the instrument. In addition to a reduction of analyte signal to noise caused by higher backgrounds, interferences may react with the analytes, causing unexpected mass shifts that will severely complicate spectral interpretation $[8,9]$. Chemical noise therefore imposes a fundamental limit on the detection of trace analytes [10]. Many obscure sources of contamination in mass spectrometry have been identified $[9,11-14]$, while still others remain to be exposed [18].

Here we report the identification of a seemingly obscure source of contamination in reversed-phase gradient HPLC and mass spectrometry. Interfering compounds are traced to nylon membrane filter disks which, ironically, are frequently employed to reduce mobile phase contaminants. MS fragmentation studies 
identified these interfering compounds as cyclic oligomers of polyamide 66 .

\section{Experimental}

\section{Materials and Reagents}

HPLC grade acetonitrile and methanol were obtained from Fisher Scientific (Ottawa, ON, Canada). Milli-Q grade water was generated on a water purification system at $18.2 \mathrm{M} \Omega \mathrm{cm}^{-1}$. The $47-\mathrm{mm}$ membrane filter disks (Millipore, Bedford, MA), all having $0.45 \mu \mathrm{m}$ pore sizes, included nylon 66, hydrophobic and hydrophilic polytetrafluoroethylene (PTFE), and mixed cellulose ester membranes. Nylon 66 filters were also purchased from Sigma-Aldrich (Oakville, ON, Canada). Trifluoroacetic acid (TFA) (98\%), formic acid (95\%), and all other chemicals used in these experiments were from SigmaAldrich.

\section{Instrumentation}

The LC system was an Agilent 1100 series LC instrument, with a diode array detector (Agilent Technologies, Palo Alto, CA). A Supelco octadecylsilyl (C18) bonded phase silica column $(15 \mathrm{~cm} \times 4.6 \mathrm{~mm}$ i.d., $5 \mu \mathrm{m}$ particles) was used in all UV absorbance experiments, while the LC/MS experiments were conducted with a C18 column (10 $\mathrm{cm} \times 1.0 \mathrm{~mm}$ i.d., $5 \mu \mathrm{m}$ particles) from Vydac (Grace Vydac, Hesperia, CA).

Mass spectra were acquired on (1) a ThermoFinnigan LCQ Duo ion trap (ThermoFinnigan, San Jose, CA), which is part of the Maritime Mass Spectrometry Laboratory at Dalhousie University, or on (2) an Agilent LC-MSD SL ion trap system (Agilent Technologies, Palo Alto, CA), located at the Institute for Marine Biosciences in Halifax, Nova Scotia. Each system was equipped with electrospray ionization (ESI), and operated in positive mode. System (1) employed a capillary temperature of $150^{\circ} \mathrm{C}$, its voltage was $4 \mathrm{~V}$, and the cone voltage was $4.2 \mathrm{kV}$. System (2) was operated with a nebulizer gas of 15 psi; dry gas flow: $5 \mathrm{~L} / \mathrm{min}$; dry temperature: $325^{\circ} \mathrm{C}$; $\mathrm{V}_{\text {cap }}$ : $-4 \mathrm{kV}$; skimmer: $40 \mathrm{~V}$; cap exit: $140 \mathrm{~V}$; oct 1: $12 \mathrm{~V}$; oct 2: $1.7 \mathrm{~V}$; trap drive: 60; ICC: $200 \mathrm{~ms}$; scan range: 200-1000. All LC/MS and LC/MS ${ }^{2}$ spectra were acquired using (1) in data dependant mode, scanning a range of $150-2000 \mathrm{~m} / \mathrm{z}$ and with a flow rate of 8 $\mu \mathrm{L} / \mathrm{min} . \mathrm{MS}^{3}$ and $\mathrm{MS}^{4}$ experiments were conducted on (2) by infusion of the sample at $5 \mu \mathrm{L} / \mathrm{min}$, in manual MS(n) mode to optimize fragmentation of each individual ion.

\section{LC Separations}

UV absorbance chromatograms were recorded at a wavelength of $214 \mathrm{~nm}$. With the $4.6 \mathrm{~mm}$ i.d. column, separations were conducted at $1 \mathrm{~mL} / \mathrm{min}$, using water as Solvent A, and acetonitrile as Solvent B. Before the start of the gradient, the column was equilibrated in
Solvent A for a specified period (see the Results section). The gradient was as follows: 0 to $50 \%$ B in a linear increase over $10 \mathrm{~min}$, then to $75 \% \mathrm{~B}$ over $4 \mathrm{~min}$. For LC/MS, $0.5 \%$ (vol/vol) formic acid was added to each solvent. The flow rate was $50 \mu \mathrm{L} / \mathrm{min}$ through the 1.0 $\mathrm{mm}$ column, and was split post-column to $8 \mu \mathrm{L} / \mathrm{min}$ for MS analysis.

\section{Experimental Procedures}

Unless otherwise stated, $1.0 \mathrm{~L}$ of the aqueous solvent was filtered through a membrane filter. The organic solvent (mobile phase B) was utilized without filtration throughout the experiments. To estimate the concentration of the contaminants, unfiltered water was spiked with $\varepsilon$-caprolactam to a concentration of $0.207 \mathrm{mg} / \mathrm{L}$. This solution was employed as Solvent A in a standard LC gradient. The area of the $\varepsilon$-caprolactam peak in the $\mathrm{UV}$ chromatogram was weighted to the number of amide functional groups per molecule. BSA $(2 \mathrm{mg} / \mathrm{mL})$ was reduced with $4 \mathrm{mM}$ dithiothreitol $\left(55^{\circ} \mathrm{C}, 20 \mathrm{~min}\right)$, alkylated with $8 \mathrm{mM}$ iodoacetamide (20 $\mathrm{min}$, room temperature), and digested with trypsin $\left(37^{\circ} \mathrm{C}, 16 \mathrm{~h}\right)$, using a 200:1 mass ratio of protein to trypsin.

\section{Results and Discussion}

During routine work, we observed two strong peaks in our UV chromatograms that were not associated with the sample. These background signals are also observed in LC/MS experiments. A base peak ion chromatogram from a typical "blank" LC/MS run is displayed in Figure 1, with the interfering peaks labeled I and II. The MS spectra corresponding to these peaks are also shown in the figure. The first eluting peak, I, displays a protonated molecular ion peak at $m / z 453.3$, whereas the

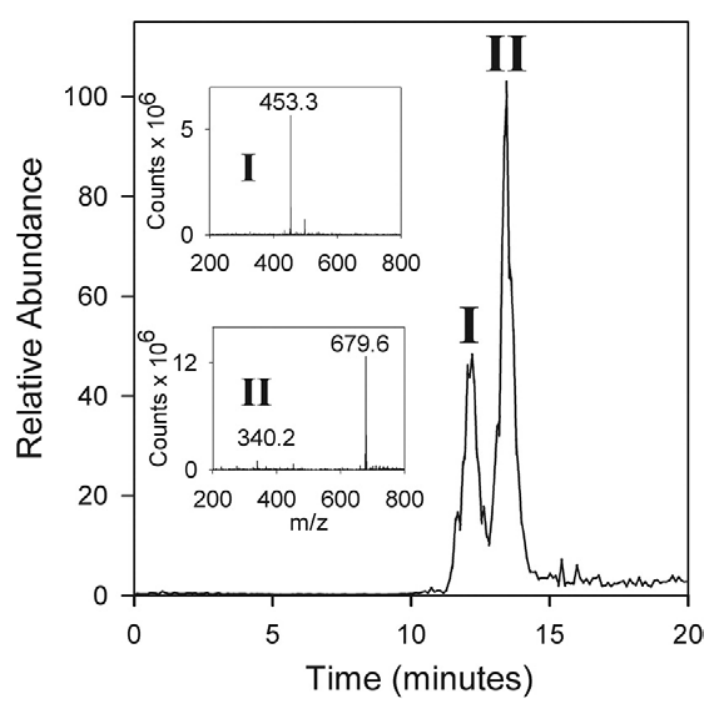

Figure 1. Base peak ion chromatogram of a blank run on a C18 column equilibrated for $30 \mathrm{~min}$ in Solvent A (water), which was filtered through a nylon 66 membrane. The MS spectra obtained for peaks I and II are also shown. 
second compound, II, has an $m / z$ of 679.6 . The areas of these peaks are directly proportional to the column re-equilibration time (30 min here), thus indicating that the contaminants are associated with the mobile phase.

It was deduced that the contaminants are associated with the filtration of the aqueous solvent. Notably, when unfiltered solvent was employed, the contaminating peaks were not observed. The source of contamination was traced to the disposable membrane disks that are commonly employed to filter particulates from the mobile phase. Filtering the mobile phase is particularly important when preparing aqueous buffers or solvents with salts, as nondissolved components can reduce column lifetimes, and lower column performance. Testing several nylon (polyamide 66) membranes from a particular manufacturer, we observed the two interfering signals at approximately equal intensity. Nylon membranes from an independent manufacturer also revealed these two interferences, although the intensity of peak I was significantly reduced. Membrane filters composed of cellulose ester, and two types of modified PTFE, a hydrophobic and a hydrophilic filter, yielded essentially "clean" chromatograms displaying only minor peaks in the blank chromatograms. These results indicate that the interferences are generic to nylon 66 membrane filters, although to different extents depending on the manufacturer.

We attempted to rinse the membranes to eliminate the extractables before collecting the solvents. Standard practice involves discarding the first few milliliters of solvent coming into contact with a new membrane. Rinsing the membranes with $250 \mathrm{~mL}$ of water before collecting the filtrate reduced the intensities of peaks I and II to $\sim 10 \%$ of their original value. Similarly, rinsing with $20 \mathrm{~mL}$ of methanol also lowered the peak intensities to $\sim 10 \%$ of their original values. However, such signals still represent significant contamination. We did not pursue larger volumes of methanol: extensive rinsing of a disposable membrane filter with methanol is neither practical nor economical.

We have observed peaks at other $\mathrm{m} / \mathrm{z}$ values associated with these interferences. In particular, when analyzing LC effluent incorporating $0.1 \%$ TFA in the solvent, a multitude of prominent signals is observed, as listed in Table 1. Through MS/MS analysis, these peaks were attributed to adducts of the interfering com-

Table 1. Nylon adducts with corresponding $m / z$ values

\begin{tabular}{lcc}
\hline \multicolumn{1}{c}{ Composition } & $m / z$ & Norm. intensity \\
\hline \hline$\left[M_{1}+H\right]^{+}$a & 453.4 & 0.28 \\
{$\left[M_{1}+K+\text { TFA }\right]^{+}$} & 605.3 & 0.11 \\
{$\left[M_{\|}+H\right]^{+}$b } & 679.5 & 0.77 \\
{$\left[M_{\|}+N a\right]^{+}$} & 701.8 & 0.23 \\
{$\left[M_{\|}+K\right]^{+}$} & 717.4 & 0.1 \\
{$\left[M_{\|}+K+\text { TFA }\right]^{+}$} & 831.5 & 0.78 \\
{$\left[M_{\|}+2 K+3 T F A-H\right]^{+}$} & 1097.3 & 1
\end{tabular}

${ }^{\mathrm{a}} \mathrm{M}_{1}$ represents the cyclic nylon dimer.

${ }^{\mathrm{b}} \mathrm{M}_{\mathrm{II}}$ represents the cyclic nylon trimer.

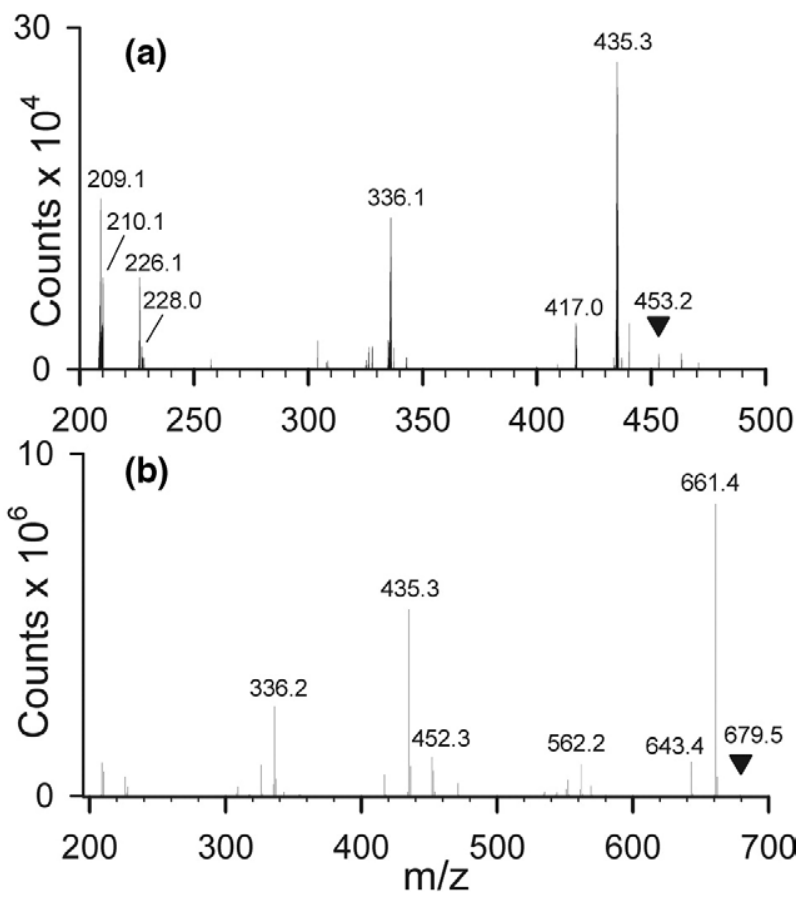

Figure 2. MS/MS spectra corresponding to (a) Compound I, with an $[\mathrm{M}+\mathrm{H}]^{+}$of $453.4 \mathrm{u}$, and (b) Compound II, with an $[\mathrm{M}+$ $\mathrm{H}^{+}$of $679.6 \mathrm{u}$.

pounds with $\mathrm{Na}^{+}, \mathrm{K}^{+}$, and TFA. It is therefore apparent that the MS spectrum of a seemingly simple contamination can quickly become much more complex as these compounds interact with other components of the sample.

\section{Identification of Interfering Compounds}

Knowing the molecular weight and origin of these compounds, it was suspected that Compounds I and II are a cyclic dimer and a cyclic trimer of polyamide 66 (molecular weights of 452.3 and $678.5 \mathrm{u}$, respectively). Nylon 66 is a linear polymer manufactured from the condensation of adipic acid with hexamethylene diamine, although cyclic oligomers are also formed as a by-product. To remove these components from the polymer, a scrubbing procedure is employed in the industrial synthesis [15]. Despite this, cyclic oligomers remain, and have frequently been observed by MS and UV, either through direct analysis of the polymer [1620], or by analyzing a solubilized portion of the polymer following solvent extraction [21-24]. The origin of interfering signals at $\mathrm{m} / \mathrm{z} 453.3,679.5$, and 905.7 have been discussed on the Association of Biomolecular Resource Facilities web-based discussion forum (www. abrf.org), and were recently attributed to nylon oligomers arising from filtering of solvents with nylon 66 membranes [25]. It is therefore evident, from our results and from others, that cyclic oligomers are present in nylon membranes, and that these compounds will migrate into aqueous solvents during the solvent filtering process. 

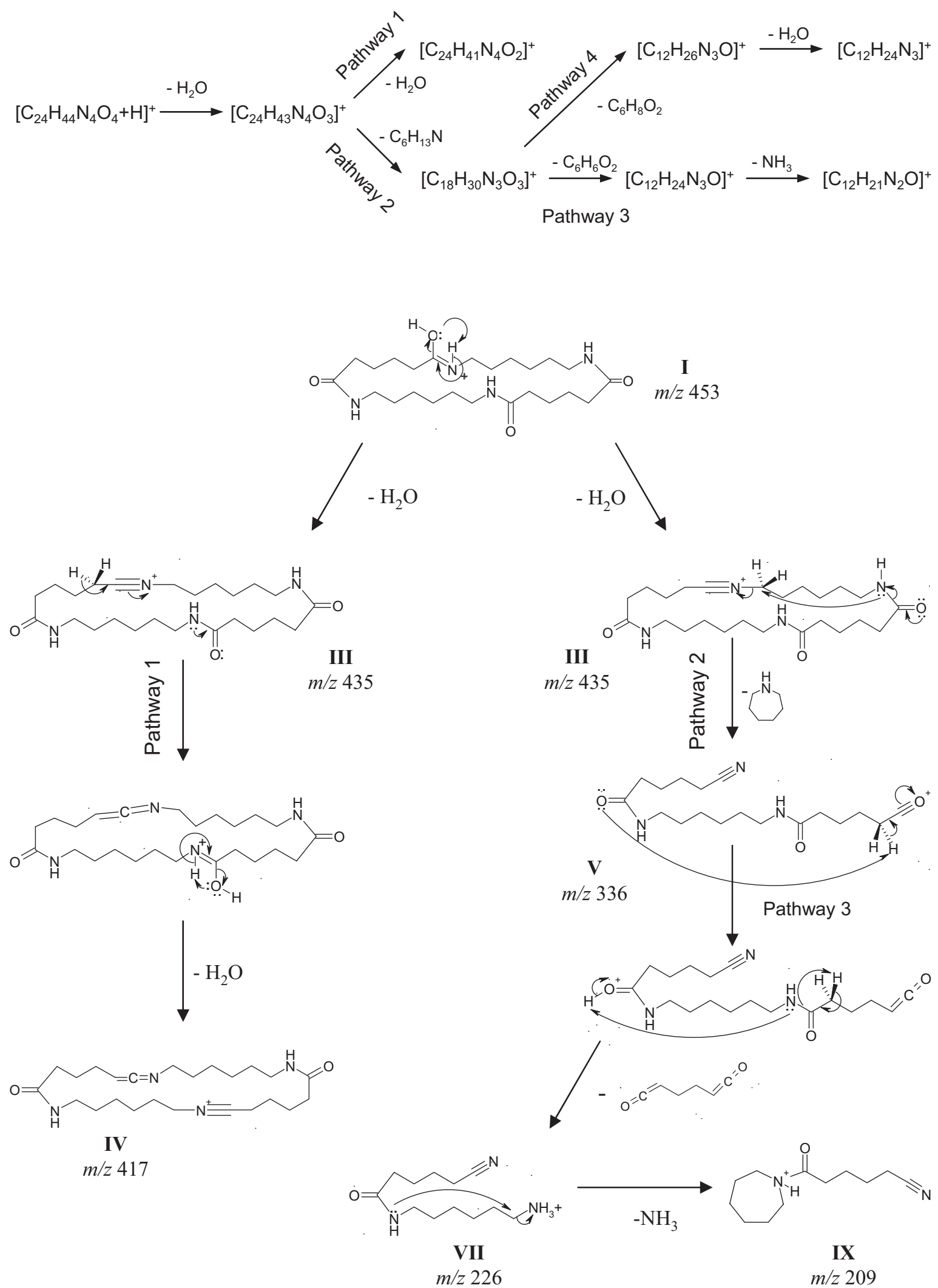

Scheme 1. Fragment ions of cyclic nylon dimer. 
The tandem-MS fragmentation spectra for I and II confirm that these two compounds indeed have very similar structures. Representative MS/MS spectra of I and II are displayed in Figure 2a and b, respectively. To determine the fragmentation pathways for the most prominent ions in the MS/MS spectrum of $\mathbf{I}, \mathrm{MS}^{3}$ and $\mathrm{MS}^{4}$ experiments were conducted. The deduced pathways are summarized in Scheme 1. Many of the fragment ions observed in the MS/MS spectrum of the ion at $\mathrm{m} / \mathrm{z} 453$ can translate to the fragment ions of the 679 peak being shifted to higher mass by $226 \mathrm{u}$ (one nylon monomer). The most prominent of these ions are labeled in Figure $2 b$. Interpretation of these fragmentation spectra provides conclusive evidence to support the identification of these compounds as cyclic oligomers of polyamide 66 .

\section{Concentration of Extractables}

Using an $\varepsilon$-caprolactam standard, the concentration of the cyclic oligomers in our mobile phase can be estimated [26]. For the contaminating signals observed in Figure 1, we calculate that $10 \mu \mathrm{g}$ of I and $24 \mu \mathrm{g}$ of II are extracted from a single nylon membrane into $1.0 \mathrm{~L}$ of water. This corresponds to about 5 and $8 \mathrm{pmol}$ of $\mathbf{I}$ and II, respectively, being detected in a single LC/MS experiment.

The intensities of the observed contamination signals are substantial. A comparison of signal intensities is provided through the analysis of $100 \mathrm{pmol}$ of a standard tryptic BSA digest, injected on column in an LC/MS experiment (16 pmol are thus directed to the mass spectrometer following the split). With $30 \mathrm{~min}$ column re-equilibration, the background signal from the nylon trimer was still the most intense signal in the ion chromatogram. The five most prominent BSA peptides observed have relative abundances ranging from 52 to $64 \%$ versus the nylon trimer. We note that the mass spectrometer employed in these experiments is an older generation system (16 pmol BSA yields $\sim 50 \%$ sequence coverage). Newer MS instruments routinely detect protein samples at significantly greater dilutions. However, in such experiments, the concentration of nylon contaminants will remain constant, giving rise to severe background signals that will almost certainly overwhelm the experiment.

It is concluded that the contaminating compounds arising from nylon membrane filters are detrimental to trace level analysis in both UV and MS detection of LC chromatograms. We recommend avoiding the use of nylon 66 membrane filters, and suggest other membranes, such as cellulose ester or PTFE, as potential alternatives in cases where filtering is deemed necessary.

\section{Acknowledgments}

The authors thank J. S. Grossert for helpful discussions related to fragmentation mechanisms, as well as R. D. Guy, L. Ramaley, and
D. A. Volmer for kindly providing access to facilities. This work was supported by the Natural Sciences and Engineering Research Council of Canada (NSERC), as well as the Department of Chemistry and the Faculty of Science at Dalhousie University.

\section{References}

1. Williams, S. Ghost Peaks in Reversed-Phase Gradient HPLC: A Review and Update. J. Chromatogr. A 2004, 1052, 1-11.

2. Bristol, D. W. Detection of Trace Organic Impurities in Binary Solvent Systems: A Solvent Purity Test. J. Chromatogr. A 1980, 188, 193-204.

3. Regnault, C.; Kano, I.; Darbouret, D.; Mabic, S. Ultrapure Water for Liquid Chromatography-Mass Spectrometry Studies. J. Chromatogr. A 2004, 1030, 289-295.

4. Hughes, D. E.; Bramer, A. M. Anomalous Liquid Chromatographic Results Originating from the Presence of Soap Residues and the Use of Nylon Filters. J. Chromatogr. A 1987, 408, 296-302.

5. Gabler, R.; Hedge, R.; Hughes, D. Degradation of High Purity Water on Storage. J. Liq. Chromatogr. 1983, 6, 2565-2570.

6. Skjevrak, I.; Due, A.; Gjerstad, K. O.; Herikstad H. Volatile Organic Components Migrating From Plastic Pipes (HDPE, PEX, and PVC) into Drinking Water. Water Res. 2003, 37, 1912-1920.

7. Junk, G. A.; Avery, M. J.; Richard, J. J. Interferences in Solid-Phase Extraction Using C-18 Bonded Porous Silica Cartridges. Anal. Chem. 1988, 60, 1347-1350.

8. Collings, B. A.; Campbell, J. M.; Mao, D.; Douglas, D. J. A Combined Linear Ion Trap Time of Flight System with Improved Performance and MS $^{n}$ Capabilities. Rapid Commun. Mass Spectrom. 2001, 15, 1777-1795.

9. Purves, R. W.; Gabrielski W.; Li, L. The Effect of Using Silicon Based Diffusion Pump Fluid on Spectral Quality in an Electrospray Ionization Ion Trap/Time-of-Flight Mass Spectrometer. Rev. Sci. Instrum. 1997, 68, 3252-3253.

10. Krutchinsky, A. N.; Chait, B. T. On the Nature of Chemical Noise in MALDI Mass Spectra. J. Am. Soc. Mass Spectrom. 2002, 13, 129-134.

11. Aebi, B.; Henion, J. D. The Reduction of Chemical Noise in an Atmospheric Pressure Ionization/Ionspray Interface for Mass Spectrometry. Rapid Commun. Mass Spectom. 1996, 10, 947-951.

12. Verge, K. M.; Agnes, G. R. Plasticizer Contamination from Vacuum System O-Rings in a Quadrupole Ion Trap Mass Spectrometer. J. Am. Soc. Mass Spectrom. 2002, 13, 901-905.

13. Xia, Y. Q.; Patel, S.; Bakhtiar, R.; Franklin, R. B.; Doss, G. A. Identification of a New Source of Interference Leached from Polypropylene Tubes in Mass-Selective Analysis. J. Am. Soc. Mass Spectrom. 2005, 16, 417-421.

14. Gibson, C. R.; Brown, C. M. Identification of Diethylene Glycol Monobutyl Ether as a Source of Contamination in an Ion Trap Mass Spectrometer. J. Am. Soc. Mass Spectrom. 2003, 14, 1247-1249.

15. Bikales, N. M. Encyclopedia of Polymer Science, Vol. X; John Wiley and Sons: New York, 1969; pp 460-482.

16. Weidner, S. M.; Just, U.; Wittke, W.; Rittig, F.; Grubber, F.; Friedrich, J. F. Analysis of Modified Polyamide 66 Using Coupled Liquid Chromatography and MALDI-TOF-Mass Spectrometry. Int. J. Mass Spectrom. 2004, $238,235-244$.

17. Ballistreri, A.; Garozzo, D.; Giuffrida, M.; Montaudo, M. Identification of the Ions Produced by Fast Atom Bombardment Mass Spectrometry in Some Polyesters and Polyamides. Anal. Chem. 1987, 59, 2024-2027.

18. Bletsos, I. V.; Hercules, D. M. Time-of-Flight Secondary Ion Mass Spectrometry of Nylons: Detection of High Mass Fragments. Anal. Chem. 1985, 57, 2384-2388.

19. Carroccio, S.; Concetto, P.; Montaudo, G. MALDI Investigation of the Photo-Oxidation of Nylon-66. Macromolecules 2004, 37, 6037-6049.

20. Mengerink, Y.; Peters, R.; van der Wal, S. Claessens, H. A.; Cramers, C. A. Endgroup-Based Separation and Quantitation of Polyamide 66 by Means of Critical Chromatography. J. Chromatogr. A 2002, 949, 337-349.

21. Porter, S. R.; Taylor, L. T. Supercritical Fluid Extraction of Nylon 66 Oligomers and their Characterization via Liquid Chromatography Coupled with Mass Spectrometry. J. Chromatogr. A 1999, 855, 715-721.

22. Ballistreri, A.; Garozzo, D.; Giuffrida, M.; Montaudo, G. Mechanism of Thermal Decomposition of Nylon 66. Macromolecules 1987, 20, 29912997.

23. Burrell, M. C.; Chao, H. S. I.; Meerman, T. P.; Peterson, G. S. Detection of Nylon 66 Cyclic Monomers on Polymer Surfaces Using Static SIMS. Surf. Interface Anal. 1997, 25, 799-803.

24. Mori, S.; Furusawa, M.; Takeuchi, T. Reduction-Gas Chromatographic Determination of Cyclic Monomer and Oligomers in Polyamides. Anal. Chem. 1970, 42, 661-662.

25. Önnerfjord, P. March 16, 2004; http://www.abrf.org/index.cfm/list. $\mathrm{msg} / 58765$.

26. Mengerink, Y.; Peters, R.; Kerkhoff, M.; Hellenbrand, J.; Omloo, H.; Andrien, J.; Vestjens, M.; van der Wal, S. Analysis of Linear and Cyclic Oligomers in Polyamide 6 Without Sample Preparation by Liquid Chromatography Using the Sandwich Injection Method. II. Methods of Detection and Quantification and Overall Long-Term Performance. J. Chromatogr. A 2000, 878, 45-55. 